\title{
Psychometric Properties of the Iranian Version of the Stages of Change Readiness and Treatment Eagerness Scale
}

\author{
Aliakbar Parvizifard ${ }^{1}$, Haji Jamludin Bin Haji Ahmad², Maznah Binti Baba² \& Tajularipin Sulaiman ${ }^{2}$ \\ ${ }^{1}$ Department of Clinical Psychology, Kermanshah University of Medical Sciences, Kermanshah, Iran \\ ${ }^{2}$ Faculty of Educational Study, Universiti Putra Malaysia, Selangor, Malaysia \\ Correspondence: Aliakbar Parvizifard, Department of Clinical Psychology, Farabi Hospital Kermanshah, Iran. \\ E-mail: aliakbar.parvizifard@gmail.com
}

Received: December 6, 2011

Accepted: January 29, 2012 Published: June 1, 2012

doi:10.5539/ijps.v4n2p246

URL: http://dx.doi.org/10.5539/ijps.v4n2p246

\begin{abstract}
The aim of the present study was to determine the psychometric properties of the Iranian version of the stages of change readiness and treatment eagerness scale, which had been translated and cross-culturally modified into Persian language and back-translated to English. Most specifically, the study sought to validate the Iranian version of the stages of change readiness and treatment eagerness scale, based on the evaluation of two experts. An additional objective was to test the translated instrument for its reliability. For this purpose, the validated instrument was administered to 80 male drug addicts, who were outpatient services at a government rehabilitation center. The results showed the both experts indicated that the items of the translated instrument had very high relevance to their present context. The inter-rater reliability of the scores given by the two experts indicated .805 between the experts' scores for the total scale.

The findings showed that the alpha coefficients for the total scale scores at pre-test and post-test were .832 and .787 , respectively. Also, the results indicated that the alpha coefficients for all the subscales of the translated instrument at pre-test ranged from .69 to .81. In all the subscales, alphas at post-test ranged from .66 to .79. The Pearson correlation test indicated a high correlation coefficient of .86 between test and retest. In short, the study showed that the translated instrument has very high content validity and high reliability and that it can be used to assess motivation for behavior change and predict treatment outcomes in drug addicts that are receiving treatments in Iran.
\end{abstract}

Keywords: opiate dependency, the stage of change theory, psychometric properties, the stages of change readiness and treatment eagerness scale, Iran

\section{Introduction}

Substance use disorders (SUDs) represent a serious public health problem all over the world (Marlatt, 2005). Opiate dependence and abuse have become a major health and social problem in Iran (Jafari, M, Shahidi, Sh, \& Abedin, 2009; Mokri, 2002). Both the amount of drug consumption and the number of drug addicts in Iran has increased since 1992. Majority of Iranian drug addicts are males in their productive years. Previous studies have shown that majority of drug addicts (46\%) in Iran began drug abuse between the ages of 17 and 22 (Madani \& Razzaghi, 2004; Mokri, 2002; Rahbar, 2003). As this age range represents the majority of the Iranian population, one of the basic priorities and major worries for the government is to find suitable methods for dealing with the problem of drug abuse among the Iranian youths (Molaie, Shahidi, Vazifeh, \& Bagherian, 2010). According to Vosooghi (2005), the Iranian drug addicts in rehabilitation centers show poor treatment compliance which leads to high rates of relapse among them. Therefore, in recent years, drug abuse treatment has been considered a major social and health problem in Iran. The high rates of relapse among Iranian male drug addicts have increased interest in assessing and enhancing motivation to change behavior of substance misuse in this population. One of the major priorities for researchers and clinicians is to develop suitable psychological instruments for assessing the motivation of behavior change and predict treatment outcomes in drug addicts that are receiving treatments in Iran. Clinicians and researchers use different approaches in assessment, diagnosis and treatment of people with substance related disorders. Bio-psychosocial approaches to treatment continue to be developed and tested. In order to determine the efficacy of these approaches for treatment of people with substance related disorders, a valid and reliable instrument that can assess the motivation and treatment outcome 
of the clients should be used. Without such instruments, the effectiveness of treatments cannot be tested. Additionally, use of unsuitable instruments may lead to results that lack of reliability and validity (Woody, Cottler, Caccola, \& Grant, 1993).

The Stage of Change Theory has received growing attention in assessment, research and treatment of drug abusers. Effective assessment of the Stage of Change behavior for people with substance use disorders is essential for clinical research, treatment planning, and referral to adjunctive services. Prochaska and DiClemente (1982a) suggested the trans-theoretical model (TTM) that describes the dynamic processes for five stages of motivational changes which can occur in individuals with drug dependence and abuse. This model offers a conceptual framework for understanding the process of change in people with drug use problems. According to Prochaska and DiClemente (1982b), the process of behavioral change is divided into five stages. These stages include pre-contemplation, contemplation, preparation, action and maintenance, respectively. Each stage is related to a specific motivational level ranging from having no plan to change (Pre-contemplation stage) to achieving significant behavioral change (Action stage) leading to an intention to keep the new behavior (Maintenance stage). According to the trans-theoretical model (TTM), in the pre-contemplation stage, the individual is unaware of negative consequences of drug use and is not thinking about starting an intervention program to change behavior. In the contemplation stage, the individual becomes aware of the problems of drug use but feels ambivalent about it. In the preparation stage, the individual knows the negative consequences of drug use and considers changing his/her drug abuse behaviors. In the action stage, the individual makes attempts to change the problematic behavior. Finally in the maintenance stage, the individual takes steps to prevent relapse into drug abuse (Prochaska, Norcross, \& DiClemente, 1995). The trans-theoretical model (TTM) is cyclical as opposed to linear; individuals can regress back to a previous stage at anytime. Matching drug treatment strategies accurately to the Stage of Change leads to significantly successful behavioral changes. Research has shown that when the drug treatment strategy is mismatched to the Stage of Change, treatment outcome is significantly less satisfactory as compared to those receiving stage-matched treatment strategies (Blissmer \& McAuley, 2002). Clinicians should be conscious that people with substance use disorders (SUDs) may feel helpless in the pre-contemplation stage due to past failures in attempting an intervention program. The original English version of the stage of change readiness and treatment-eagerness scale (the SOCRATES) was designed by Miller and Tonigan (1996) to assess motivation for behavior change in people with substance use disorders. The SOCRATES is an assessment instrument which can be used to assess motivation for behavior change and predict treatment outcomes among clinical populations (DiClemente, Nidecker, \& Bellack, 2008; DiClemente, Schlundt, \& Gemmell, 2004; Freyer et al., 2005; Miller \& Tonigan, 1996). The SOCRATES consists 19 statements about changing drug using behavior on 5 points Likert scale that ranged from strongly disagree (1) to strongly agree (5). Subjects were asked to rate their answers to each item on the scale using the rating criteria of strongly disagree, disagree, less agree, agree and strongly agree. The SOCRATES has a possible score range from 19 to 95 . The SOCRATES has three subscales namely, Recognition (7.items; identification substance use as problem), Ambivalence (4.items; uncertainty about one's decision to change substance use) and taking steps (8.items; behaviors taken to change substance use). The scores from each item in the subscales are added for subscale scores. Summation of all item's scores to gather yielded the total of scale. Previous studies have investigated psychometric properties of the original SOCRATES among participants with drinking problem (Burrow-Sanchez \& Lundberg, 2007; Figlie, Dunn, \& Laranjeira, 2005; Maisto et al., 1999; Miller \& Tonigan, 1996). Given the lack of a translation of the SOCRATES in Iran, the researchers translated the instrument into Persian using an exhaustive procedure to achieve a satisfactory cross-cultural equivalence. The same instrument designed for a particular assessment context should be validated before being used in a different context. Psychological tests are often used in different cultural settings without detailed attention to the cross-cultural variations. In adapting and/or translating psychological instruments from a language to another the conceptual, semantic, and technical equivalence between the original version of instruments and the target language should be closely considered. The aim of this study was, therefore, to investigate the psychometric properties of the Iranian version of the stages of change readiness and treatment eagerness scale (the SOCRATES-IV) among Iranian drug addicts at a government rehabilitation center. The following research questions were posed to meet the objectives of the study:

1) How do experts evaluate the SOCRATES-IV in reference to the relevance of its items to their present testing context?

2) What is the level of reliability of the translated version of the instrument?

The next section discusses the method that was followed to provide answers for these research questions. 


\section{Method}

\subsection{Participants}

This study was conducted at a government rehabilitation center in Kermanshah, Iran. The sample consisted of 80 male drug addicts consecutively admitted to outpatient services in the rehabilitation center. All the participants met the diagnostic criteria in the statistical manual of mental disorders (Association \& DSM-IV., 2000) for opiate dependence, as confirmed by the structured clinical interview diagnostic (SCID-1) based on DSM-IV conducted by a psychiatrist. The participants were assessed three times (diagnostic assessment, test, and retest). The study was approved by the ethical committee of Kermanshah University of Medical Sciences (KUMS) after the participants had consented to participate in the study. The average age of the participants was 35.86 years $(\mathrm{SD}=5.98$, range $25-45)$. More than half $(56.25 \%)$ of the participants were married. Most of them $(62.5 \%)$ had high school level education and three in four were employed. The average of drug use history of the subjects was 11 years $(\mathrm{SD}=7.5)$. As for the types of drugs used, opium (thariac, shireh and sukhte) was the most commonly drug $(57.5 \%)$, followed by heroin $(22.5 \%)$ and hashish (20.\%). The main route of administration of drug use was by smoking $(56.25 \%)$, followed by oral (22.5\%) and inject (21.25\%) (Table 1$)$.

Table 1. Socio-demographic characteristics of the participants of study $(\mathrm{N}=80)$

\begin{tabular}{ccc}
\hline Characteristics & $\mathbf{N}$ & $\mathbf{\%}$ \\
\hline Range of age (25-45) & 80 & 100 \\
$(\mathrm{M}=35.86$ years, SD $=5.95)$ & - & - \\
Married status: & 45 & 56.25 \\
Married & 35 & 43.75 \\
Single/ Divorced & & \\
Education : & 50 & 62.5 \\
High school level & 30 & 37.5 \\
College level & 60 & 75 \\
Occupation status & 20 & 25 \\
Employment & & \\
unemployment & 46 & 57.5 \\
Types of Drug Used: & 18 & 22.5 \\
Opium (thariac, Shireh, Sukhte) & 16 & 20 \\
Heroin & & \\
Hashish & 45 & 56.25 \\
Smoke & 18 & 22.5 \\
Oral & 17 & 21.25 \\
Inject & & \\
Administration of Drug Used: & & \\
\hline & & \\
\hline
\end{tabular}

\subsection{Instruments}

The demographic data, the Iranian version of the SCID-I and the SOCRATES-IV were used in this study. The SCID-I is a semi-structured interview for screening axis-I disorders by DSM-IV. According to Sharifi et al. (2009), the SCID-I yields diagnoses with acceptable to good reliability and validity in a clinical population in Iran. The SOCRATES-IV consists 19-statements about changing drug using behavior on 5 points Likert scale that ranged from strongly disagree (1) to strongly agree (5). The SOCRATES-IV has three subscales namely, Recognition (7-items), Ambivalence (4-items) and taking-steps (8-items). The scores from each item in the subscales are added for the subscale scores. Summation of all item's scores to gather yielded the total of scale. The SOCRATES-IV has a possible score range from 19 to 95 . The original SOCRATES reliability of recognition is .85- 95; the ambivalence is .60-88; and the taken step is .83-96 (Miller \& Tonigan, 1996). This scale is the 
public domain instrument and would be used without special permission.

\subsection{Procedure}

In this study, the original Stage of Change Readiness and Treatment Eagerness Scale (the SOCRATES) were translated from English to Persian. Then, two bilingual experts who read and spoke both Persian and English languages evaluated the translated SOCRATES for content validity. The experts had different academic degrees such as $\mathrm{PhD}$ in clinical psychology and $\mathrm{PhD}$ in psychiatry rehabilitation. Based on the experts' view, sentences recognized as weakly worded or unclear were rewritten by the researcher. Also, the back translation technique was used in order to attain a culturally equivalent instrument. To sum up, the study consisted of five steps as described below:

1) The original SOCRATES was translated from English to Persian language, considering the suggestion made by the experts.

2) Two experts were asked to translate back the SOCRATES-IV from Persian to English.

3) The back translated instrument was reviewed by the researchers to cross-check the content and meaning of each item between the back translated instruments (English) and the original instruments (English).

4) The SOCRATES-IV was revised by the researchers based on the feedback and recommendations from the two experts.

5) The final revision of the SOCRATES-IV was used to determine its psychometric properties.

In order to determine the psychometric properties of the SOCRATES-IV, it was administered to 80 participants who were being treated for drug use at a government rehabilitation center in Iran. Before gathering data, the participants were given a copy of the instrument and were briefed on the research objectives and the scoring scale in the SOCRATES-IV. The instrument was administered to the participants individually. The participants were asked to rate their responses to each statement on the scale using the rating criteria of strongly disagree, disagree, less agree, agree and strongly agree. After the participants rated all the statements of the questionnaire (which took approximately 60 minutes), the researchers enquired the participants about their understanding of each statement in order to indentify which, if any, were confusing. Finally, the psychometric properties of the SOCRATES-IV were analyzed.

\subsection{Data Analysis}

In this study, Cronbach's alpha and Pearson correlation methods were used to assess the internal consistency, the inter-rater reliability between the experts' scores, and test-retest reliability scores of the SOCRATES-IV, respectively.

\section{Results and Discussion}

As mentioned earlier, to determine the content validity of the SOCRATES-IV, two experts evaluated its items regarding their relevance. For each item, the experts used a 4-point Likert scale interpreted in the following way:

1) "Absolutely irrelevant" indicated as 1 ,

2) "Irrelevant" indicated as 2 ,

3) "Relevant" indicated as 3 ,

4) "Absolutely relevant" indicated as 4.

The experts were each given a copy of the instrument along with a cover letter, providing them guidance on how to evaluate the SOCRATES-IV. There were 19 items in the instrument; therefore, the score that each expert assigned would range between 19 and 76. Table 2 shows the scores given by the two experts: The percentage of the scores was calculated and the results were interpreted based on Guilford's rule of thumb, which regards a value of $>20 \%$ as Negligible, $20-40 \%$ as Low, $40-70 \%$ as Moderate, $70-90 \%$ as High and $90 \%$ as Very high level of relevance (Guilford, 1950). As the results show, both experts indicated that the items had very high relevance to their present context. In order to check the inter-rater reliability of the scores given by the two experts, Pearson Product Moment Coefficient test was used. The results showed a moderate correlation (.725) between the scores assigned by the two experts. According to Farhadi, Jafarpur and Birjandi (2001), a correlation coefficient of below .50 is regarded as low, .50 to .75 as moderate and .75 to .90 as high. Additionally, Cronbach's alpha was used to test reliability of the instrument. The results showed that the Cronbach's alpha Coefficients for the total SOCRATES-IV scores at pre-test and post-test was .832 and .787 , respectively. Based on Farhadi, et al. (2002), these coefficients indicate moderate to high levels of internal reliability. Also, the results indicated that the alpha Coefficients for all the subscales of the SOCRATES-IV at pre-test ranged 
from .698 to .811 . All the subscale's alpha at post-test ranged from .658 to .794 (see Table 3). Also, the Pearson correlation coefficient was calculated to examine the reliability of the SOCRATES-IV, based on the scores marked by the participants. The results showed that test-retest reliability for the SOCRATES-IV was .86 over four weeks. According to Farhadi et al. (2002), a reliability coefficient of more than .75 is considered a high indicator of consistency between the scores. Therefore, the results showed that the Iranian version of the instrument clearly had high reliability. In short, the results confirm that the Iranian version of the SOCRATES has very high content validity and high reliability and it can be used to measure what it is supposed to measure. The results of this study are consistent with those of studies that have shown good to excellent reliability for the psychometric properties of the original SOCRATES (Miller \& Tonigan, 1996; Yeh, 2009). Additionally, this value was higher than that of the reliability of the instrument as reported in previous studies, ranging from .60 to .80 (Carmines \& Zeller, 1979; Jamaludin Ahmad. \& Asyraf., 2011). Finally, this study confirmed that the Iranian version of the SOCRATES is a valid and reliable self-report instrument for the assessment of changing motivation in the addicts that are receiving treatments in the rehabilitation centers in Iran.

Table 2. Scores assigned to the Iranian version of the SOCRATES

\begin{tabular}{cccc}
\hline Expert & $\begin{array}{c}\text { Score } \\
(19-76)\end{array}$ & Percentage (\%) & $\begin{array}{c}\text { Interpretation } \\
\text { (based on the Guilford's rule of thumb) }\end{array}$ \\
\hline Psychologist & 71 & 93.42 & Very high relevance \\
Psychiatrist & 73 & 96.05 & Very high relevance \\
\hline
\end{tabular}

Table 3. The internal consistency reliability for Iranian versions of the total SOCRATES and the subscales

\begin{tabular}{|c|c|c|c|c|c|}
\hline \multirow[b]{2}{*}{ Scale } & \multicolumn{3}{|c|}{ Pre-test } & \multicolumn{2}{|c|}{ Post-test (after 4-weeks) } \\
\hline & $\begin{array}{l}\mathrm{N} . \\
\text { items }\end{array}$ & Alpha & $\begin{array}{c}\text { Standardized item } \\
\text { alpha }\end{array}$ & Alpha & $\begin{array}{c}\text { Standardized item } \\
\text { alpha }\end{array}$ \\
\hline $\begin{array}{l}\text { The total } \\
\text { SOCRATES }\end{array}$ & 19 & 0.812 & 0.835 & 0.852 & 0.791 \\
\hline \multicolumn{6}{|l|}{ The subscales : } \\
\hline Recognition & 7 & 0.792 & 0.796 & 0.766 & 0.754 \\
\hline Ambivalence & 4 & 0.811 & 0.814 & 0.794 & 0.783 \\
\hline Taking-steps & 8 & 0.694 & 0.648 & 0.663 & 0.665 \\
\hline
\end{tabular}

\section{Strengths, Limitations and Suggestions}

This study had its own strengths and limitations. It was the first study to translate the SOCRATES to Persian language for the assessment of motivation for behavior change and predict treatment outcomes in drug addicts that are receiving treatments in Iran. However, there were some weaknesses; for example, small sample size, clinical sample and lack of gender variable were some limitations in this study that limit its generalizability to other populations. As the present study has studied the psychometric properties of the SOCRATES-IV only on male drug addicts at a government rehabilitation center, thus it is suggested that a similar study can be done in different clinical settings, on more diverse populations and on female and male addicts in private rehabilitation centers. Since the present study has confirmed the SOCRATES-IV as a valid and reliable instrument, it is suggested that this instrument be used for assessing treatment outcomes and predicting motivation for change in the addicts that are receiving treatments in the rehabilitation centers.

\section{Conclusion}

The present study translated and validated the original SOCRATES into Persian language with a methodology to achieve precise cross-cultural equivalence. The results of psychometric analysis showed that the SOCRATES-IV has a very high content validity, based on two experts' evaluation (research question 1). It was also found that the new version of the instrument has moderate to high levels of reliability (research question 2). Two experts helped the researchers to assess the relevance of the items in the Persian version of the instrument. Based on the 
findings, both experts rated the SOCRATES-IV as highly valid. Therefore, it can confidently be used for measuring the motivation of readiness for change in addicts that are receiving treatments in the rehabilitation centers in Iran. The internal reliability for the SOCRATES-IV was established by calculating the Cronbach's alpha coefficients. The results of the study indicated a high alpha coefficient value (.83) for the SOCRATES-IV. Also, the study showed that test-retest reliability for the SOCRATES-IV was .86 over four weeks.

\section{References}

Association, A. P., \& DSM-IV., A. P. A. T. F. O. (2000). Diagnostic and statistical manual of mental disorders. DSM-IV-TR: American Psychiatric Publishing, Inc.

Blissmer, B., \& McAuley, E. (2002). Testing the requirements of stages of physical activity among adults: the comparative effectiveness of stage-matched, mismatched, standard care, and control interventions. Annals of Behavioral Medicine, 24(3), 181-189.

Burrow-Sanchez, J. J., \& Lundberg, K. J. (2007). Readiness to change in adults waiting for publicly funded substance abuse treatment. Addictive behaviors, 32(1), 199-204.

Carmines, E. G., \& Zeller, R. A. (1979). Reliability and validity assessment (Vol. 17). Sage Publications, Inc.

DiClemente, C. C., Nidecker, M., \& Bellack, A. S. (2008). Motivation and the stages of change among individuals with severe mental illness and substance abuse disorders. Journal of Substance Abuse Treatment, 34(1), 25-35.

DiClemente, C. C., Schlundt, D., \& Gemmell, L. (2004). Readiness and stages of change in addiction treatment. American Journal on Addictions, 13(2), 103-119.

Figlie, N., Dunn, J., \& Laranjeira, R. (2005). Motivation for change in alcohol dependent outpatients from Brazil. Addictive behaviors, 30(1), 159-165.

Freyer, J., Tonigan, J. S., Keller, S., Rumpf, H. J., John, U., \& Hapke, U. (2005). Readiness for change and readiness for help-seeking: A composite assessment of client motivation. Alcohol and Alcoholism, 40(6), 540-544.

Jafari, M, Shahidi, Sh., \& Abedin. (2009). Comparing the effectiveness of Cognitive Behavioral Therapy and Trans-theoretical Model on improving abstinence self-efficacy in substance dependent adolescents. Journal of Research in Behavioural Sciences, 1(7), 1-12.

Jamaludin Ahmad., \& Asyraf., C. A. (2011). The Construction, Validity, Reliability and Effectiveness of Drug Rehabilitation Module on Self-Concept of Female Addicts and Motivation Achievement of Male Addicts in Malaysia. International Journal of Humanities and Social Sciences, 1(10), 217-228.

Madani, S., \& Razzaghi, O. (2004). Investigating change patterns in drug use in Iran. Paper presented at the 1st National Congress on Social Issues, Tehran University, Iran.

Maisto, S. A., Conigliaro, J., McNeil, M., Kraemer, K., O'Connor, M., \& Kelley, M. E. (1999). Factor structure of the SOCRATES in a sample of primary care patients. Addictive behaviors, 24(6), 879-892.

Marlatt, G. A. (2005). Relapse prevention: Maintenance strategies in the treatment of addictive behaviors. New York: The Guilford Press.

Miller, W. R., \& Tonigan, J. S. (1996). Assessing drinkers' motivations for change: The Stages of Change Readiness and Treatment Eagerness Scale (SOCRATES). Psychology of Addictive Behaviors, 10(2), 81-89.

Mokri, A. (2002). Brief overview of the status of drug abuse in Iran. Arch Iranian Med, 5(3), 184-190.

Molaie, A., Shahidi, S., Vazifeh, S., \& Bagherian, S. (2010). Comparing the effectiveness of cognitive behavioral therapy and movie therapy on improving abstinence self-efficacy in Iranian substance dependent adolescents. Procedia-Social and Behavioral Sciences, 5, 1180-1184.

Prochaska, J. O., \& DiClemente, C. C. (1982a). Transtheoretical therapy: Toward a more integrative model of change. Psychotherapy: Theory, Research \& Practice, 19(3), 276.

Prochaska, J. O., \& DiClemente, C. C. (1982b). Transtheoretical therapy: Toward a more integrative model of change. Psychotherapy: Theory, Research \& Practice, 19(3), 276-288.

Prochaska, J. O., Norcross, J. C., \& DiClemente, C. C. (1995). Changing for good. New York: Harper Paperbacks.

Rahbar, S. (2003). Research on Social Factors Contributing to Addiction in Hamedan [in Persian], MA Thesis, 
Islamic Azad University, Northern Branch, Tehran Iran.

Sharifi, V., Assadi, S. M., Mohammadi, M. R., Amini, H., Kaviani, H., Semnani, Y., \& Shooshtari, M. H. (2009). A persian translation of the structured clinical interview for diagnostic and statistical manual of mental disorders: psychometric properties. Comprehensive psychiatry, 50(1), 86-91.

Vosooghi, M. (2005). Treatment of Addicts in a Tehran Drug Rehabilitation Center. Critique: Critical Middle Eastern Studies, 14(1), 89-99.

Woody, G. E., Cottler, L. B., Caccola, J., \& Grant, B. (1993). Severity of dependence: data from the DSM - IV field trials. Addiction, 88(11), 1573-1579.

Yeh, M. Y. (2009). A psychometric evaluation of the Chinese version of the stage of change - readiness and treatment - eagerness scale. Journal of clinical nursing, 18(17), 2494-2502. 